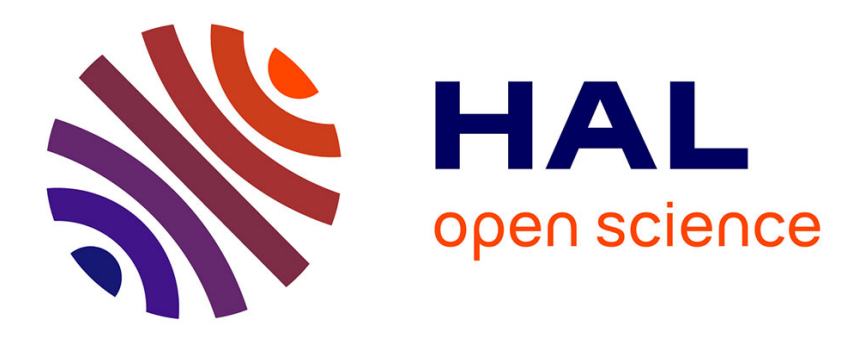

\title{
Precision linearity studies of the ATLAS liquid argon EM calorimeter
}

\author{
G. Graziani
}

\section{To cite this version:}

G. Graziani. Precision linearity studies of the ATLAS liquid argon EM calorimeter. International Conference on Calorimetry in High Energy Physics - CALOR2004 11, Mar 2004, Perugia, Italy. pp.391397. in2p3-00021997

\section{HAL Id: in2p3-00021997 https://hal.in2p3.fr/in2p3-00021997}

Submitted on 30 Jun 2004

HAL is a multi-disciplinary open access archive for the deposit and dissemination of scientific research documents, whether they are published or not. The documents may come from teaching and research institutions in France or abroad, or from public or private research centers.
L'archive ouverte pluridisciplinaire HAL, est destinée au dépôt et à la diffusion de documents scientifiques de niveau recherche, publiés ou non, émanant des établissements d'enseignement et de recherche français ou étrangers, des laboratoires publics ou privés. 


\section{Precision linearity studies of the ATLAS liquid Argon EM calorimeter}

Giacomo Graziani (LAL, Orsay)

presently at DAPNIA/CEA, Saclay

for the ATLAS LAr EM Group

XI International Conference in Calorimetry in High Energy Physics Perugia, Italy

Avril 1st, 2004 
$\Leftrightarrow \quad$ Electromagnetic Calorimetry for the general-purpose ATLAS experiment at the LHC should provide a linearity well within $1 \%$ from the $\mathrm{GeV}$ to the $\mathrm{TeV}$ scale and even much better in limited energy ranges

e.g. precision measurement of $\mathrm{W}$ mass requires a linearity within a few $10^{-4}$ between 30 and $80 \mathrm{GeV}$

$\Rightarrow \quad$ First precision linearity measurement has been performed on a LAr Barrel Calorimeter module, exposed to electron beam (CERN H8 test line)

- Need to know the beam energy scale to a few $10^{-4}$ $\Longrightarrow$ calibration of the beam line between 10 and $180 \mathrm{GeV}$

- Need to understand in principle the most suitable energy reconstruction procedure

$\Longrightarrow$ study detector response through accurate MC (Geant4) simulation

- Need to accurately inter-calibrate the detector layers $\Longrightarrow$ understand effects on linearity of calibration, noise... 


\section{Beam Energy Measurement}

$E_{\text {beam }} \propto \int B d l$

Energy range:

10 to $180 \mathrm{GeV}$

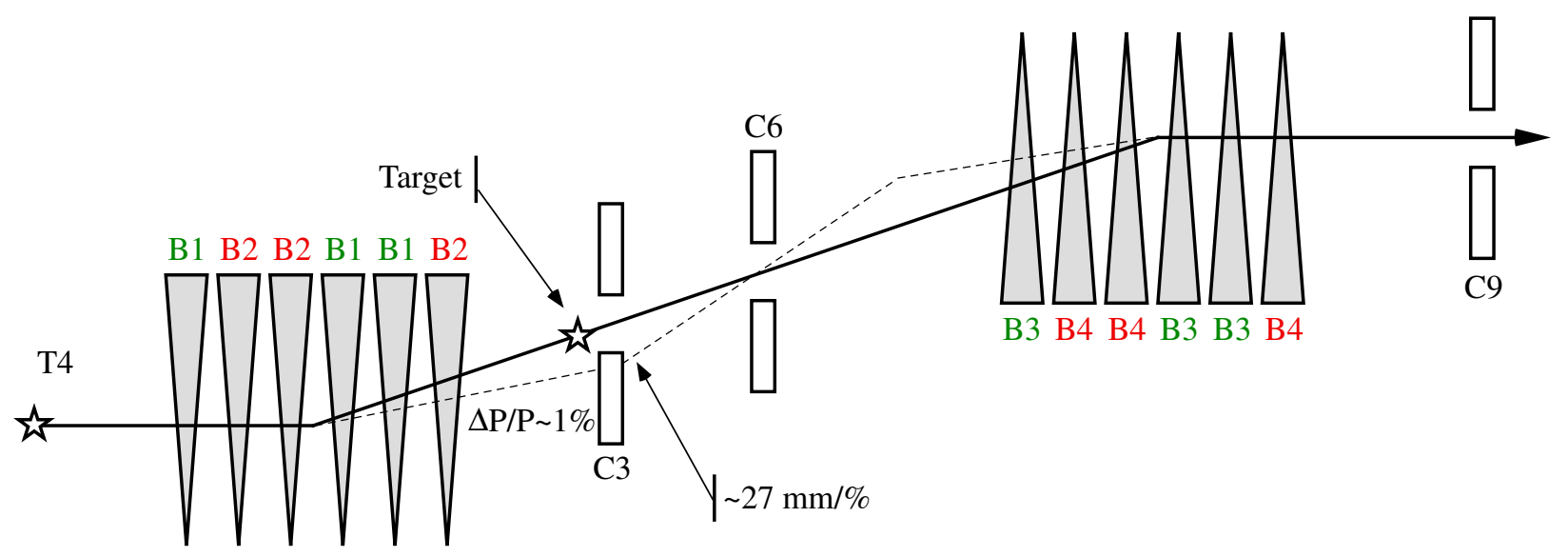

Need precise bending power calibration of a magnet triplets (B3 in the sketch, B4 off and degaussed), as a function of the current pulsed in the magnets.

The current is measured with $10^{-4}$ accuracy using DCCT.

Calibration performed by CERN SL team on a reference magnet by measuring the voltage induced by current pulsing on a narrow loop made of two wires stretched along the beam path. Accuracy: better than $10^{-4}$ on the range of interest.

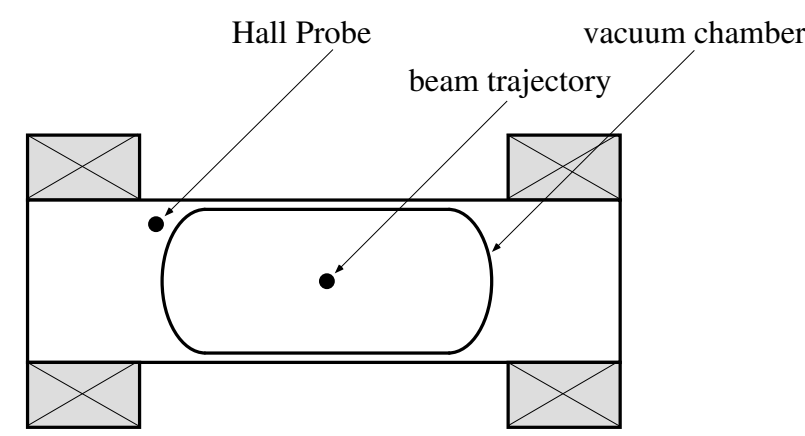

Cross-check: all along the data taking, the field in one of the B3 magnets was monitored using two Hall probes located just outside the beam pipe, in order to check the reliability of the current measurement.

The Hall probes were also calibrated on the reference magnet. 
The magnets in the test beam line and the calibration one are part of a set of 100 SPS magnets, tuned to be identical at $\pm 210^{-4}$ in 1977.

Some residual differences between calibration and beam magnets have been corrected...
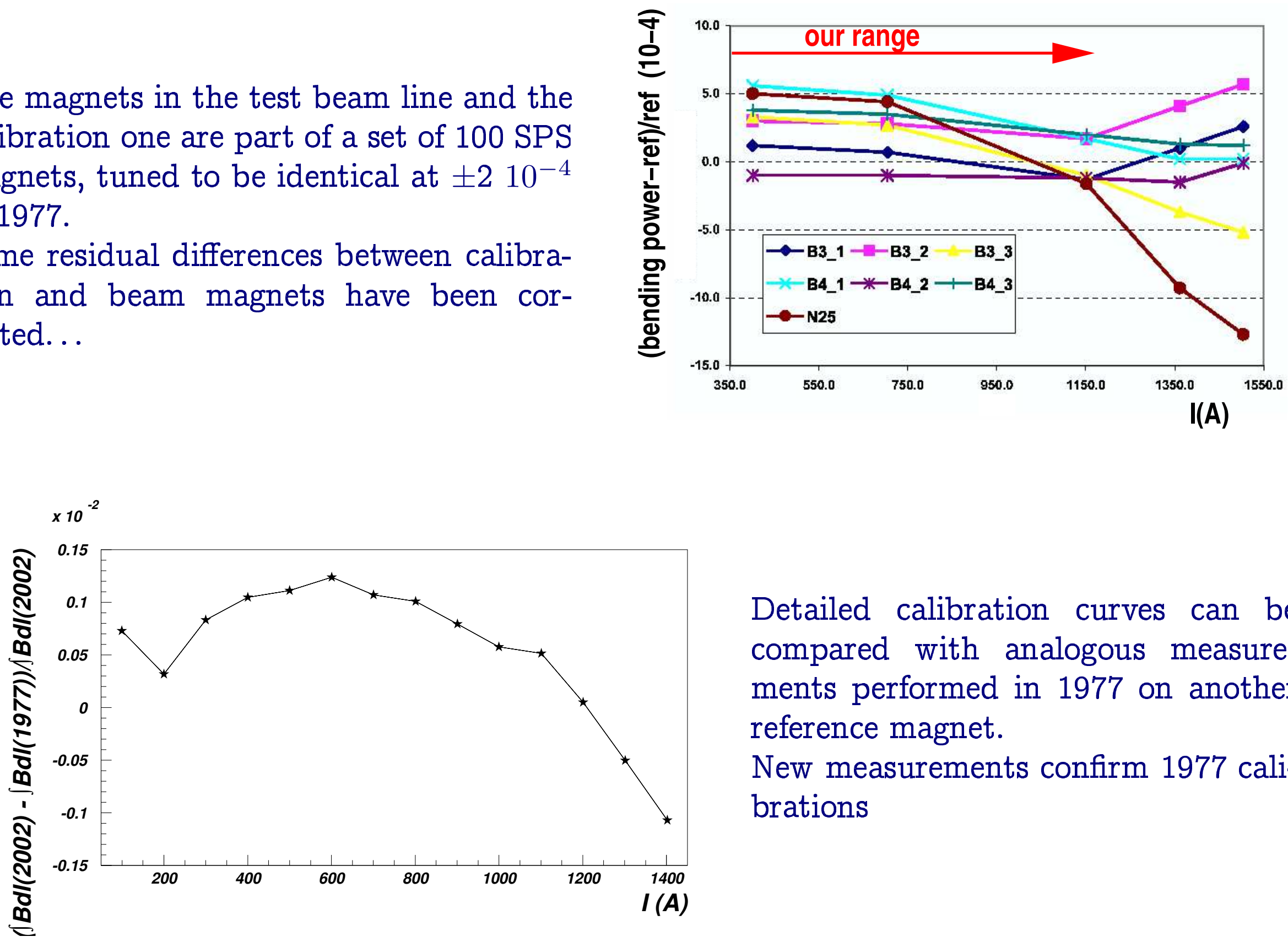

Detailed calibration curves can be compared with analogous measurements performed in 1977 on another reference magnet.

New measurements confirm 1977 calibrations 


\section{Cross-check with Hall probes}

check of absolute value of field vs current limited by differences among magnets in the border region (some discrepancies observed at the $110^{-3}$ level).

However, stability with time of measured field for a given current value within probe accuracy (few $10^{-4}$ )

$\Longrightarrow$ accuracy of current measurement validated

\section{Systematics}

The dominant effect is the residual field in the degaussed magnets, inducing an

offset on energy scale of $\pm 11 \mathrm{MeV}$

$\Longrightarrow \pm 1 \times 10^{-3}$ on linearity for $\mathrm{E}>10 \mathrm{GeV}$

Uncorrelated errors on single points (dominated by uncertainty on differences among magnets) can affect the linearity only up to $\pm 3 \times 10^{-4}$

\begin{tabular}{|r|r|r|r|}
\hline $\begin{array}{r}\text { current } \\
(\mathrm{A})\end{array}$ & $\begin{array}{r}\int B d l \\
(\mathrm{Tm})\end{array}$ & $\begin{array}{r}\text { electron mean } \\
\text { energy }(\mathrm{GeV})\end{array}$ & $\begin{array}{r}\text { error } \\
(\mathrm{GeV})\end{array}$ \\
\hline 62.315 & 1.3664 & 10.0820 & 0.0132 \\
93.133 & 2.0379 & 15.0368 & 0.0088 \\
124.363 & 2.7184 & 20.0579 & 0.0095 \\
155.513 & 3.3980 & 25.0714 & 0.0108 \\
186.689 & 4.0789 & 30.0954 & 0.0117 \\
217.900 & 4.7607 & 35.1258 & 0.0129 \\
248.973 & 5.4397 & 40.1346 & 0.0141 \\
311.270 & 6.8005 & 50.1721 & 0.0168 \\
373.484 & 8.1596 & 60.1955 & 0.0195 \\
435.705 & 9.5187 & 70.2143 & 0.0223 \\
497.673 & 10.8715 & 80.1832 & 0.0253 \\
559.524 & 12.2208 & 90.1200 & 0.0281 \\
621.128 & 13.5634 & 100.0000 & 0.0328 \\
744.113 & 16.2383 & 119.6596 & 0.0368 \\
926.708 & 20.1866 & 148.5927 & 0.0461 \\
1115.230 & 24.1095 & 177.1730 & 0.0561 \\
\hline
\end{tabular}




\section{Effect of Bremsstrahlung}

- Some material (vacuum windows, scintillation and Cerenkov counters, etc.) is spread along the $\sim 170 \mathrm{~m}$ long beam line. Bremsstrahlung photons emitted far from the detector can be lost

$\Longrightarrow$ expect a tail in the beam energy distribution

- Not easy to predict the equivalent amount of material producing the effect, expect between 0.06 and $0.1 X_{0}$

- but we can fit the reconstructed energy distributions (after a tight cut against pion contamination): tails are well reproduced at all energies with a MC simulation including Brem. losses in $0.08 X_{0}$

- tails, convoluted with the resolution, produce a bias on the peak between $0.45 \%$ $(10 \mathrm{GeV})$ and $0.12 \%(180 \mathrm{GeV})$ that has to be corrected

\section{Reconstructed energy}
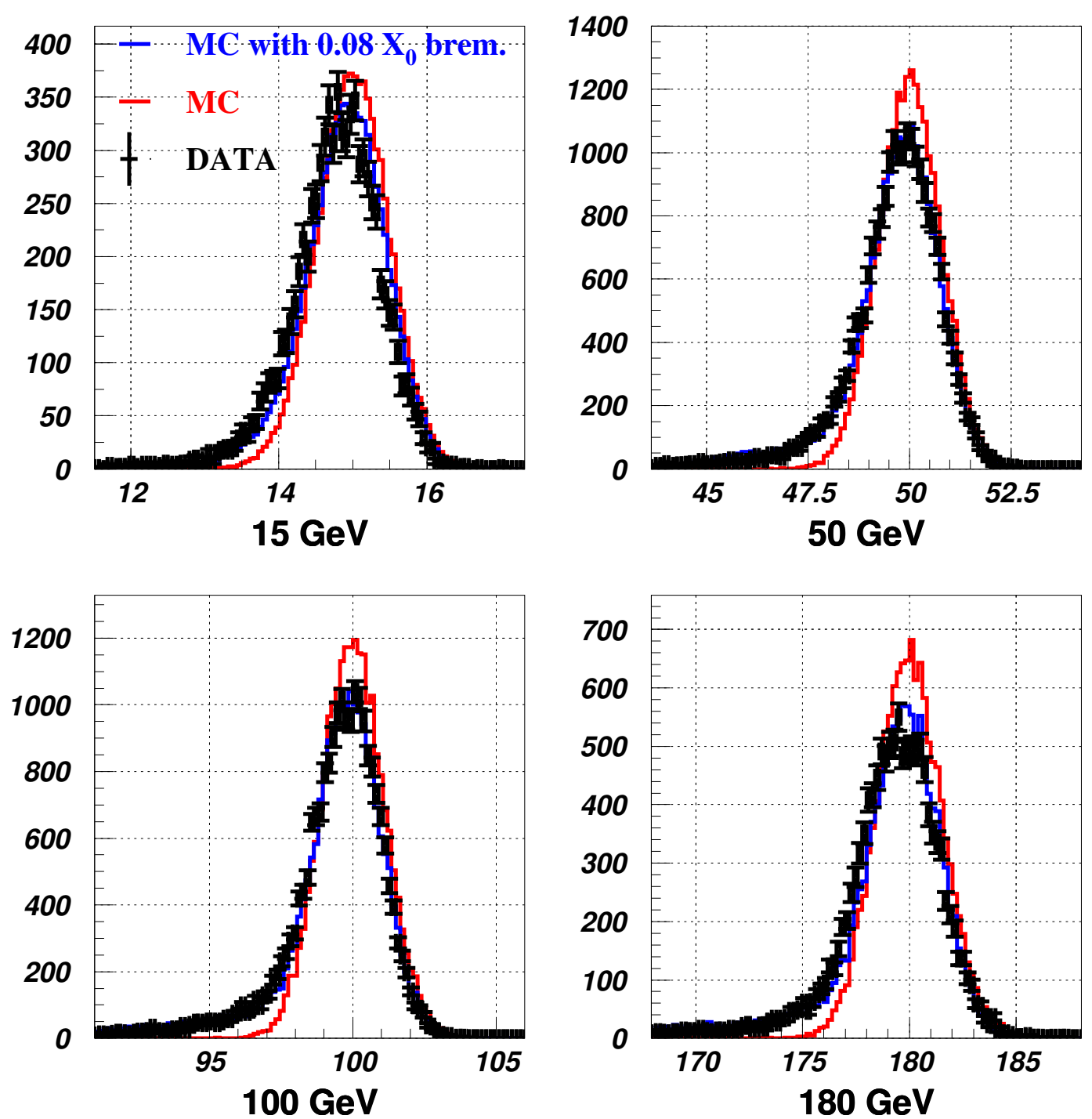


\section{The ATLAS LAr Barrel Calorimeter}

(see talks by C. de la Taille, L. Serin, O. Gaumer)

- $\mathrm{Pb} / \mathrm{LAr}$ sampling calorimeter with accordion geometry

- 4 longitudinal samplings with different transverse segmentation:

Presampler (PS)

+three accordion layers:

STRIPS, MIDDLE, BACK


$$
\eta=\mathbf{0 . 6 8 7}
$$$$
\eta=1.475
$$

energy scan at this position 
- Energy must be reconstructed from the 4 samplings

$$
E=\sum_{i=1}^{4} w_{i} E_{i}
$$

- to preserve linearity, weights should be the inverse of the sampling fraction $f_{s}$

$$
w_{i}=\frac{1}{f_{s}}=\frac{E_{\text {active }}^{e}+E_{\text {passive }}^{e}}{E_{\text {active }}^{e}}
$$

than can in principle depend on energy and can be obtained from MC:

- Full e.-m. shower Geant4 simulation, cutoff 30 or $15 \mu \mathrm{m}$

- Detector geometry in testbeam setup described in detail

- sampling fraction for electrons depends strongly on depth (Lead/Ar calorimeter!)

- accordion detector is designed carefully to have a depth-independent $f_{s}$ for mips

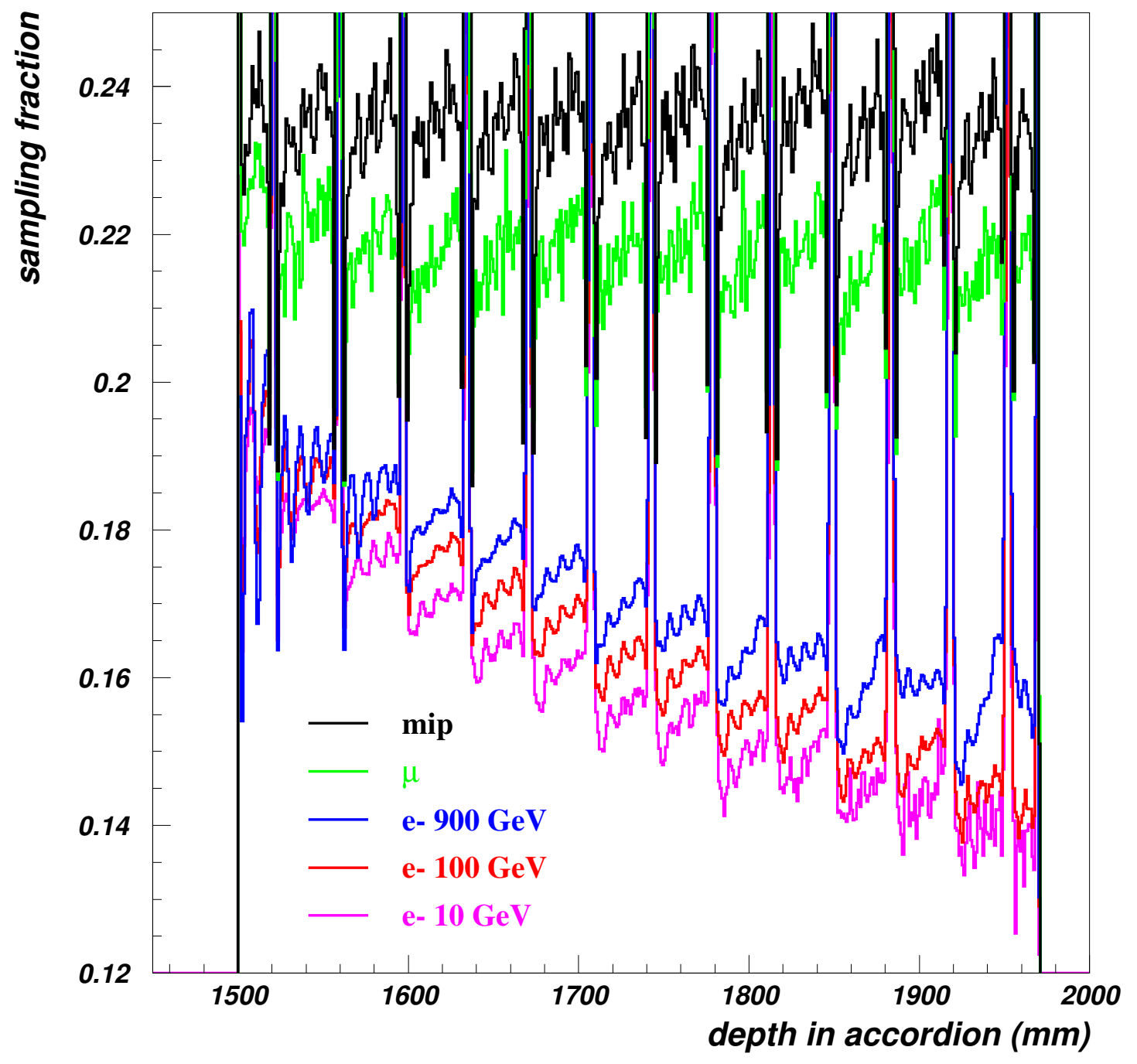


- Thus, sampling fraction scales with the longitudinal shower development

- For showers fully contained in the accordion detector, the sampling fraction becomes independent on energy and on longitudinal fluctuations

$\Longrightarrow$ need accurate intercalibration of the three accordion layers
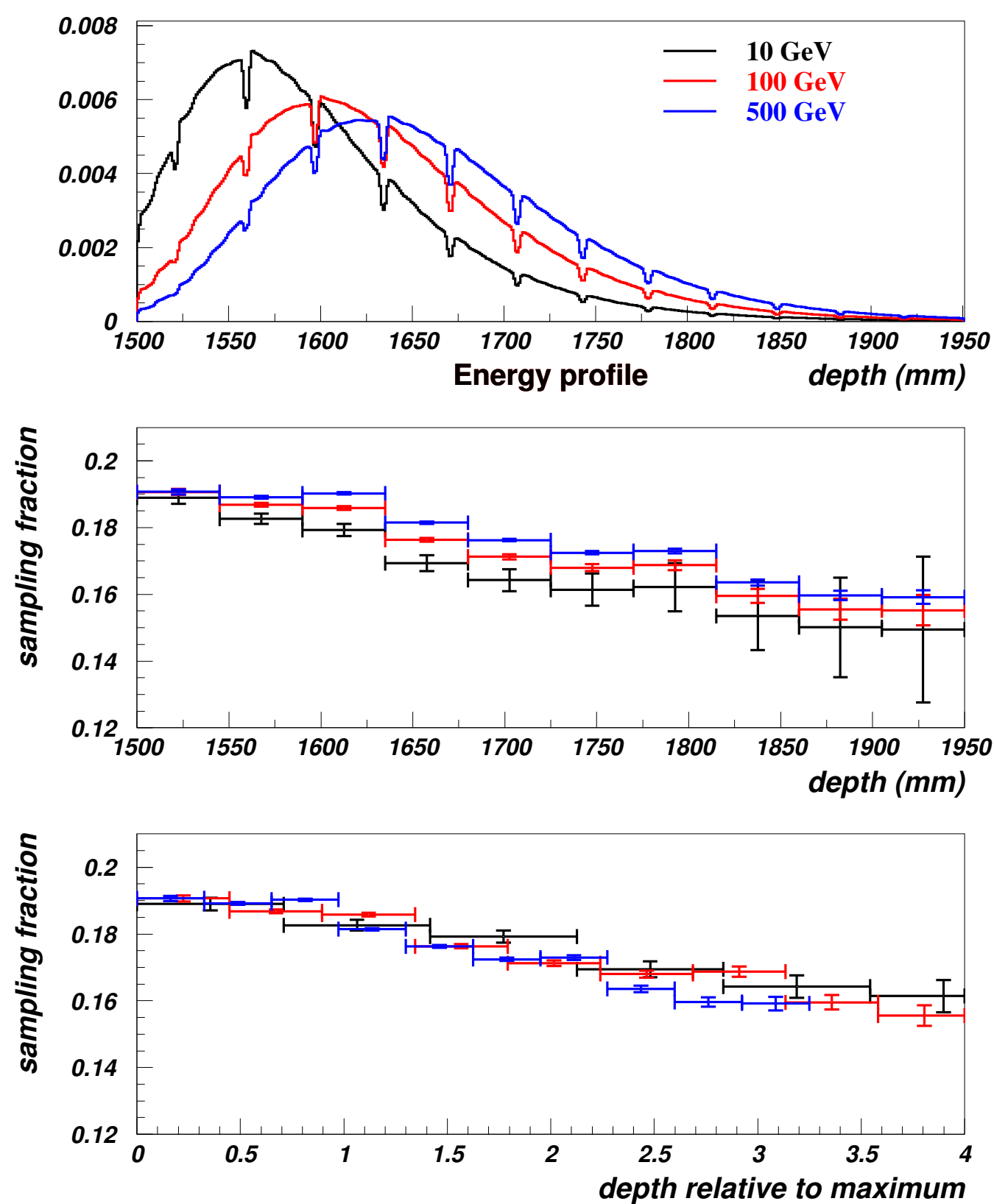
- However, some energy of the shower is lost before (passive material, PS) and after the accordion (long. leakage)

$\Longrightarrow$ residual dependence of accordion sampling fraction on event depth (estimated from longitudinal shower barycenter)

... but practically energy independent!!

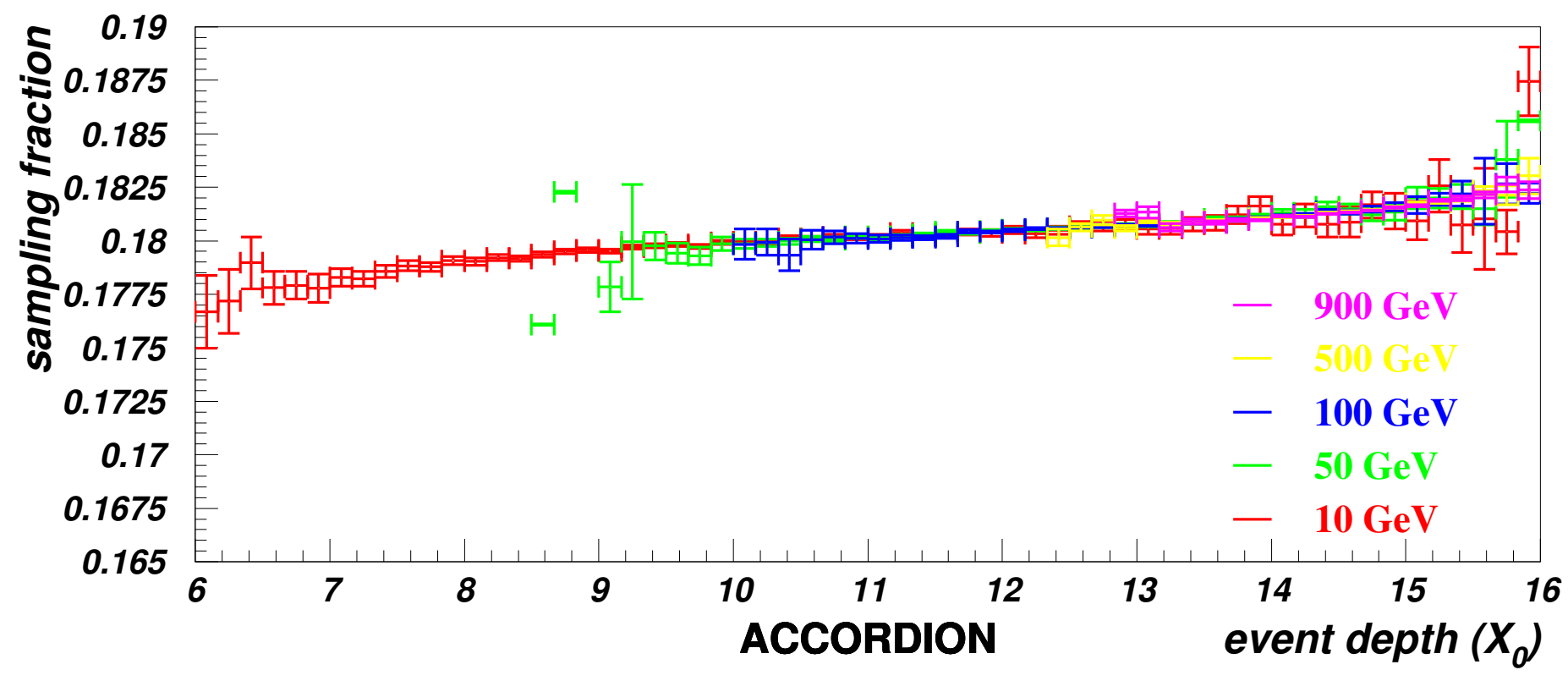




\section{Presampler calibration}

- The energy lost upstream the accordion is estimated event by event by the Presampler

- One can find empirically a "magic" weight that makes the full detector linear, and makes the reconstructed energy independent on long. fluctuations at the same time:

$$
E \propto\left(5.6 E_{P S}+E_{A C C O R D}\right)
$$

- unfortunately, this weight enhances sampling fluctuations in the PS

$\Longrightarrow$ resolution is degraded
REAL DATA
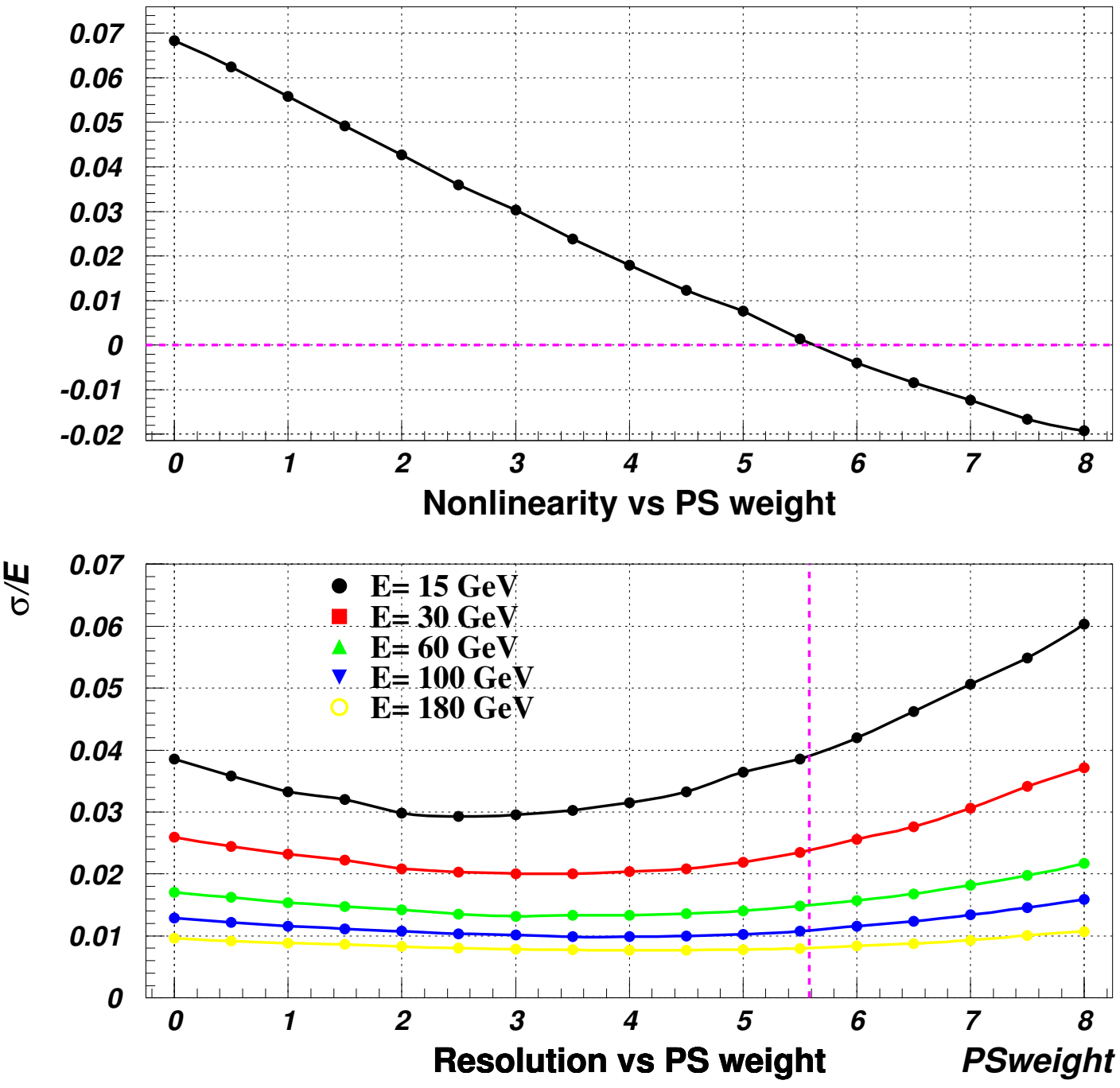
๑ Alternative: reconstruct energy as

$$
E=\frac{E_{P S}}{f_{s}^{e, P S}}+\frac{E_{A C C O R D}}{f_{s}^{e, A C C O R D}}+F_{\text {leakage }}
$$

$\Longrightarrow$ preserves the linearity by definition and gives much better resolution

- Reconstruction becomes specific for electrons

- $f_{s}^{e, A C C O R D}$ and long. leakage fraction $F_{\text {leakage }}$ can be parametrized only as a function of the event depth $D$ and $\eta$

- resolution performances depend critically on how $f_{S}^{e, P S}$ is parametrized and how the passive material between PS and STRIPS $\left(\sim 0.2 X_{0}\right)$ is taken into account;

๑ present approach: share the material between $f_{s}^{e, P S}$ and $f_{s}^{e, A C C O R D}$ and parametrize both as a function of $\mathrm{D}, \mathrm{E}$ and $\eta$

๑ under development (to improve resolution):

- upstream energy better described by $a+b E_{P S}$

- apply ad-hoc correction for the passive material $\left(\propto \sqrt{E_{P S} E_{S T R I P S}}\right)$

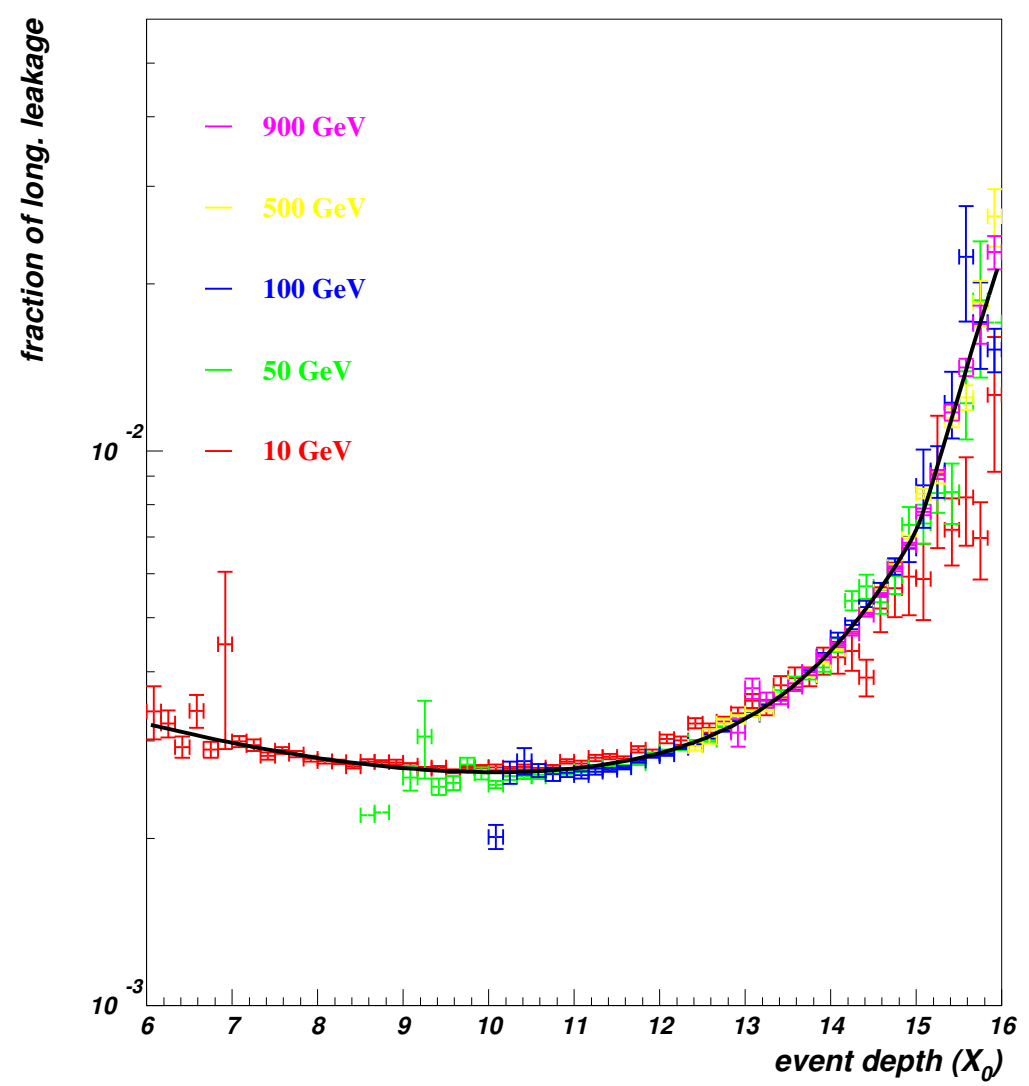




\section{DATA / MC comparison}

( $\mathrm{MC}$ includes uncoherent noise)
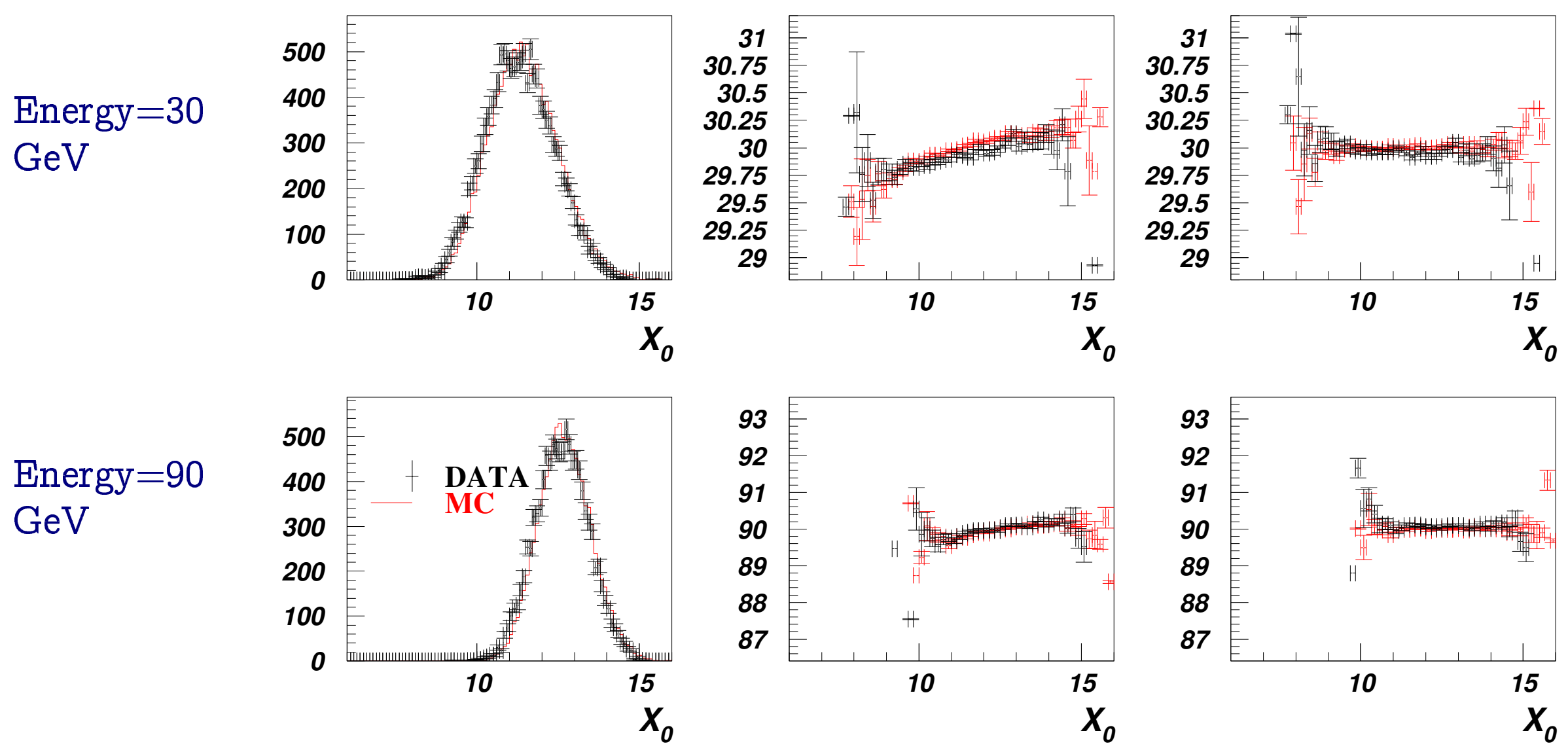

Longitudinal

Profile

Raw rec. Energy vs Event Depth $\frac{E_{P S}}{f_{s}^{e, P S}}+\frac{E_{A C C O R D}}{f_{s}^{e, A C C O R D}}$
Final rec. Energy vs Event Depth

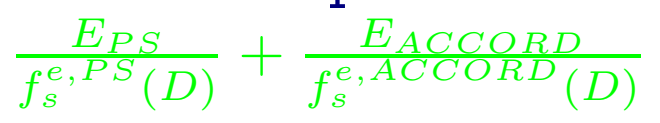


- Data taken during August 2002 at CERN H8 beam test line

- 16 energy points between 10 and $180 \mathrm{GeV}$

- only one position (beam time is limited!)

- energy reconstructed applying the weights obtained from MC simulation

- some other effects that may affect linearity, not accounted by the simulation, need to be considered:

- cell's electronic calibrations

- cross-talk and noise

- beam shape (geometrical corrections)

- pion contamination 


\section{Readout non-linearity}

- ADC dynamic range is covered by three gains (low, medium, high) differential non-linearities up to $0.5 \%$ observed in electronic calibration for medium gain

- after refining calibration procedure, the energy measured with the two gains in the gain switch region $\left(E_{M I D D L E}^{\text {cell }} \sim 20 \mathrm{GeV}\right)$ agree typically within $110^{-3}$

All channels layer MIDDLE

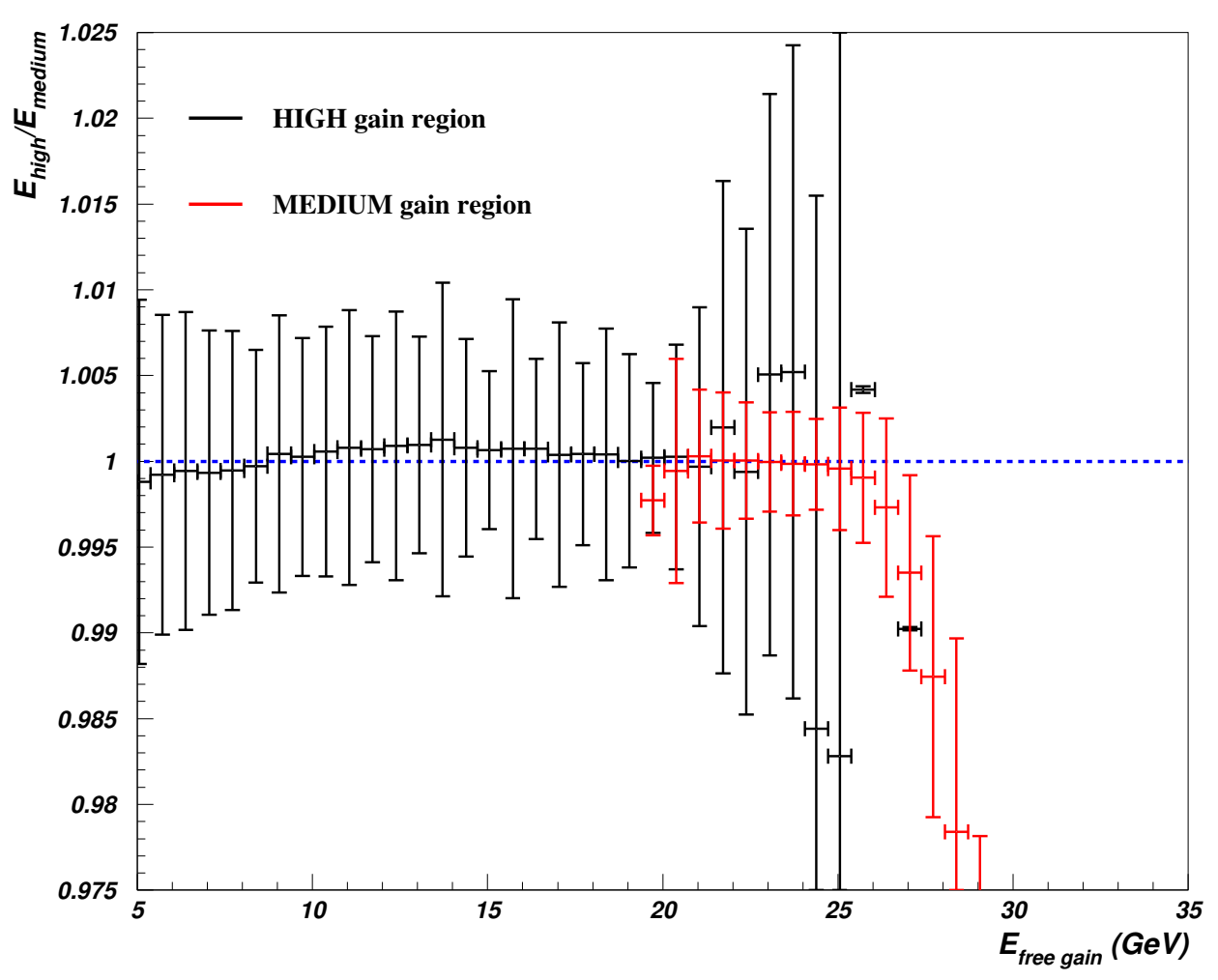




\section{Pion Contamination}

- most of pions in the beam vetoed by counter downstream the detector

- transverse profile analysis: parametrize e. $-\mathrm{m}$. shower profile with real electrons, and apply a $\chi^{2}$ cut:

$\chi^{2}=\frac{1}{N-3} \sum_{\text {cells }}^{\text {layer=1,3 }} \frac{\left(E_{i} / E_{\text {layer }}-f_{l}(\Delta \eta, \Delta \phi)\right)^{2}}{\sigma_{f_{l}}^{2}(\Delta \eta, \Delta \phi)}$

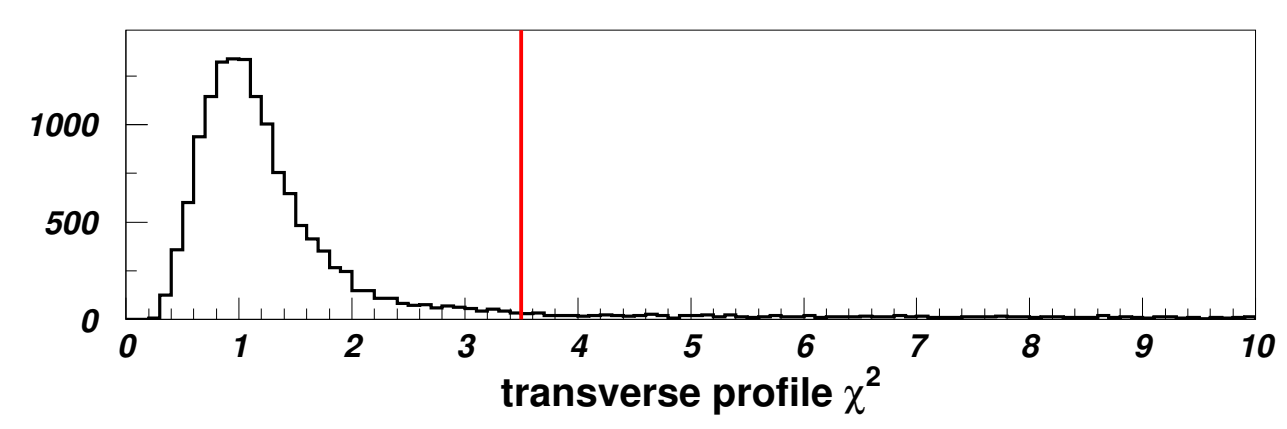

where $E_{i} / E_{\text {layer }}$ is the fraction of layer energy in cell $i$

$\Longrightarrow$ cut only depends on shower shape, not on absolute energies

- clear suppression of pions when looking at the total energy and PS energy
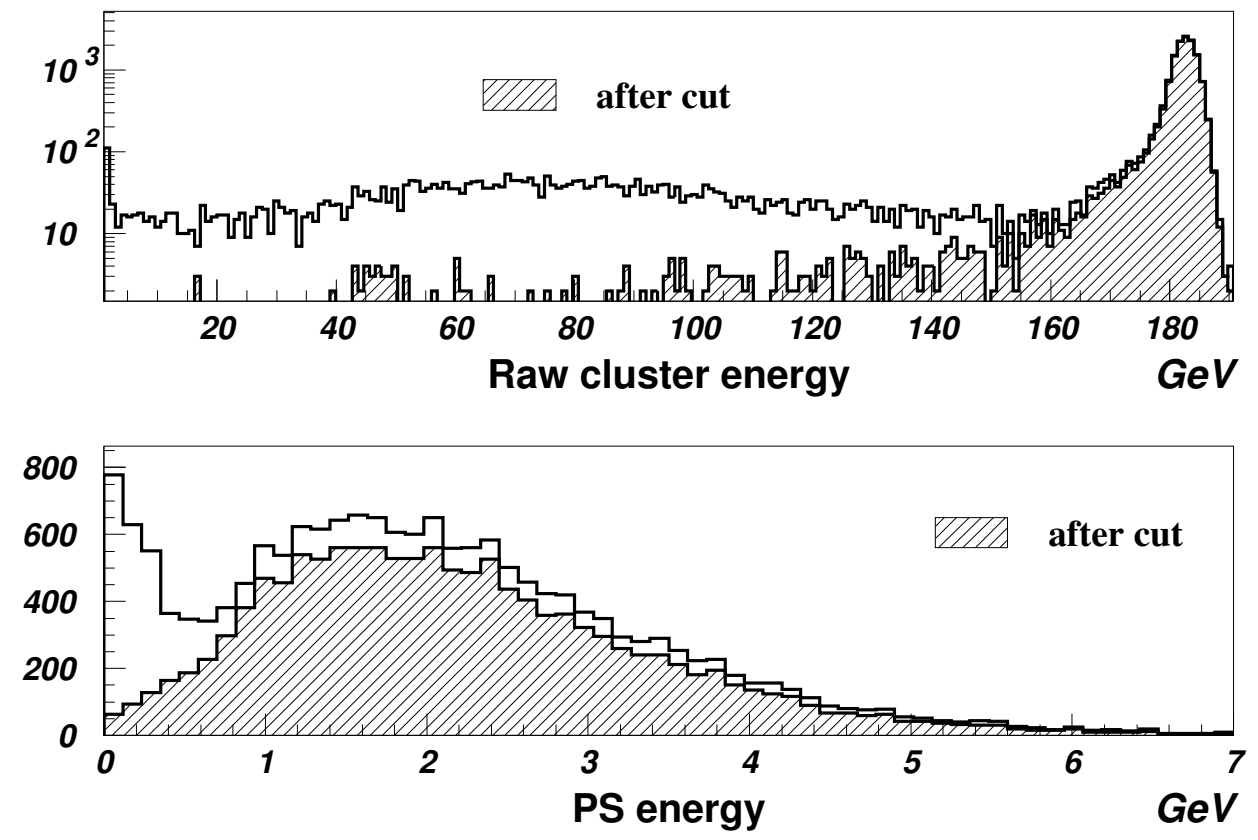


\section{Layer Intercalibration}

- guaranteed in principle by electronic calibration

- but cross-talk effects are different in each layer, due to different transverse segmentation

อ in particular, $\mathrm{a} \sim 10 \%$ cross-talk among neighbour strips (at signal peak) needs to be corrected

๑ not easy to predict the effect after signal reconstruction based on optimal filtering, present estimate $6.5 \pm 1 \%$

\section{Systematic summary}

Correlated errors:

Error on linearity for $\mathrm{E}>10 \mathrm{GeV}$

Strips cross-talk

Uncertainty on $\mathrm{MC}$ weights

$\pm 0.2 \%$

$\pm 0.15 \%$

(estimated by comparing weights obtained with

different $\mathrm{MC}$ productions, after varying the amount of passive material within uncertainty)

Beam energy offset

$\pm 0.1 \%$

Uncorrelated errors: geometrical corrections

residual pion contamination

Error on each point

$\pm 0.05 \%$

$\pm 0.05 \%$ 


\section{Results}

\section{(preliminary)}

Linearity

(normalized to $100 \mathrm{GeV}$ )

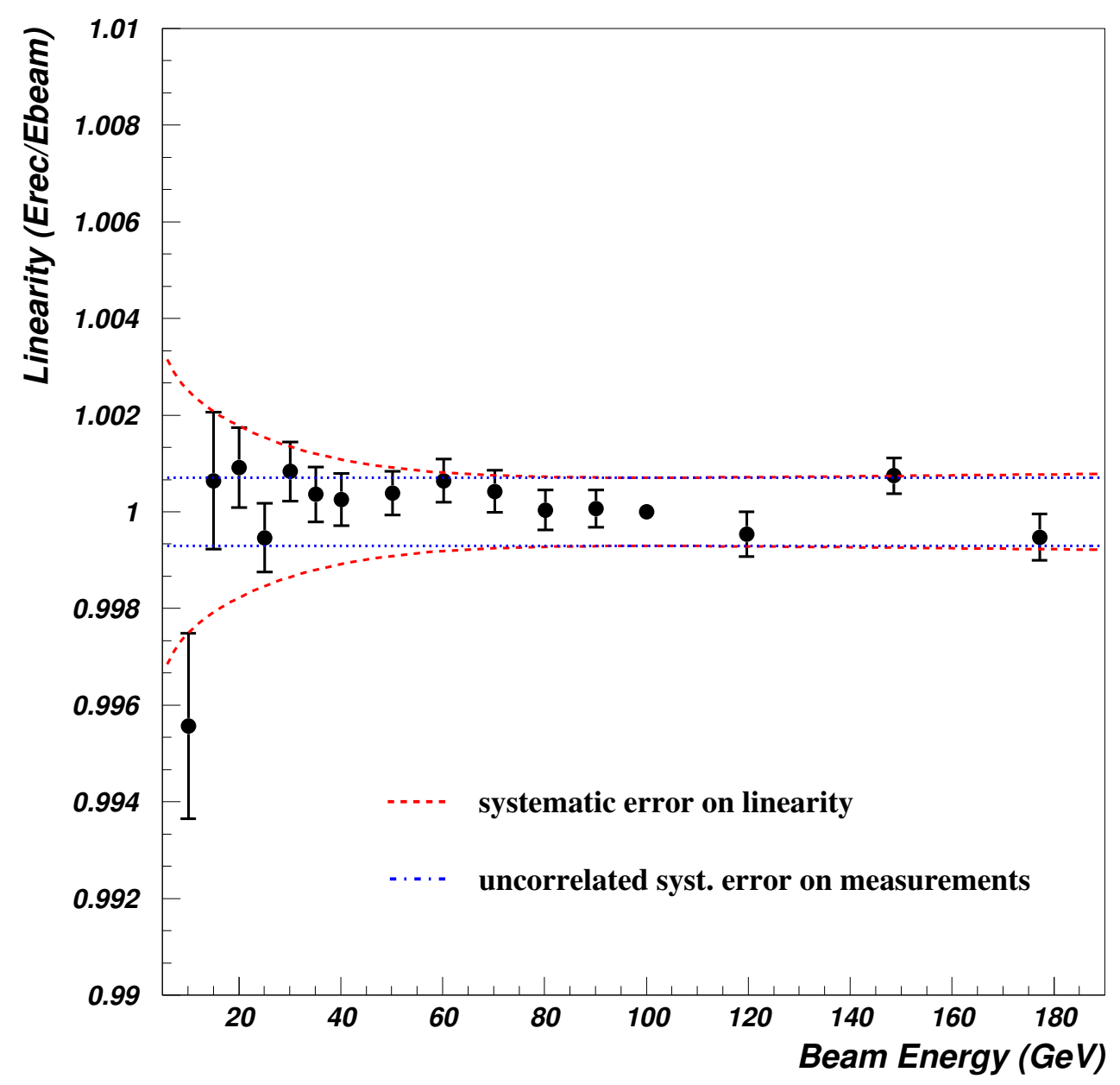

\section{Resolution}

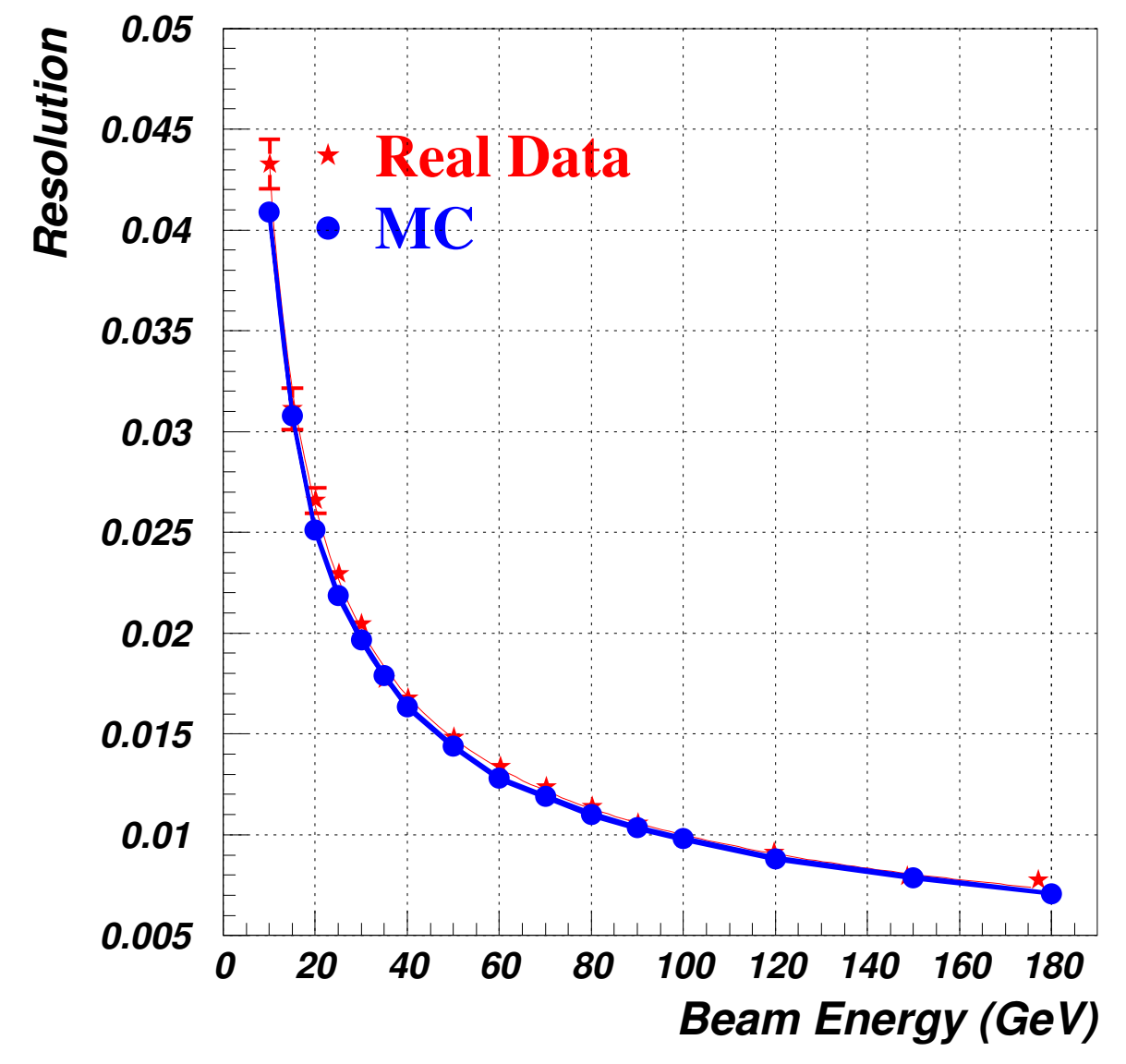


- first precision linearity study of ATLAS LAr Barrel Calorimeter

- single position scanned in the range 10 to $180 \mathrm{GeV}$

- detector was proved to be linear within $\pm 0.25 \%$ for $\mathrm{E}>10 \mathrm{GeV}$ and within $\pm 0.1 \%$ for $\mathrm{E} \gtrsim 40 \mathrm{GeV}$

- many effects investigated for the first time at this level of accuracy, still space for improvements for:

- energy reconstruction

- impact of cross-talk, noise, signal reconstruction on linearity

- new data will be collected during combined test-beam in 2004 